\title{
Gene-Editing: Interpretation of Current Law and Legal Policy
}

\author{
†a-Kyoung Kim \\ College of Law, Sungshin University, Seoul 02844, Korea
}

\begin{abstract}
With the development of the third-generation gene scissors, CRISPR-Cas9, concerns are being raised about ethical and social repercussions of the new gene-editing technology. In this situation, this article explores the legislation and interpretation of the positive laws in South Korea. The BioAct does not specify and regulate 'gene editing' itself. However, assuming that genetic editing is used in the process of research and treatment, we can look to the specific details of the regulations for research on humans as well as gene therapy research in order to see how genetic editing is regulated under the BioAct. BioAct differentiates the regulation between (born) humans and embryos etc. and the regulation differ entirely in the manner and scope. Moreover, due to the fact that gene therapy products are regarded as drugs, they fall under different regulations. The Korean Pharmacopoeia Act put stringent sanctions on clinical trials for gene therapy products and the official Notification "Approval and Examination Regulations for Biological Products, etc." by Food and Drug Safety Administration may be applied to gene editing for gene therapy purposes.
\end{abstract}

Key words : Gene-editing, Gene therapy, Gene scissors, Biosafety and bioethics act, Legislation of gene editing, Gene therapy product

\section{INTRODUCTION}

With the development of the third-generation gene scissors, CRISPR-Cas9, new Gene-Editing technologies and clinical medicines are rapidly developing in Korea. For example, researchers recently conducted a genetically modified cell therapy study in Korea, which differentiated pluripotent stem cells of hemophilia patients into hepatocytes by reprogramming the hemophiliac somatic cells and transplanting these hepatocytes into the patient's liver (Ministry, 2015). Following the enactment of Article 14 of the Framework Act on Science and Technology in 2015, the Ministry of Science, ICT and Future Planning conducted a 'Technology Impact Assessment' to evaluate the impact of new development in science and technology on the economy, society, culture, ethics, and environment. The assessment revealed that by 2019, the market for genetic editing is expected to reach $\$ 1.6$ billion (Ministry, 2015), and research is underway to apply genetic scissors to treat diseases such as AIDS and anemia in various countries around the world. In this situation it is very important to clarify how Korean laws regulate gene editing or how the present legislation is to be interpreted regarding gene editing.

\section{THE REGULATION OF KOREAN BIOACT (I): CASE OF (BORN) HUMANS}

The most important law in Korea that contains regula-

\footnotetext{
Manuscript received September 17, 2017, Received in revised form September 19, 2017, Accepted September 21, 2017

${ }^{\dagger}$ Corresponding Author : Na-Kyoung Kim, College of Law, Sungshin University, Seoul 02844, Korea. Tel: +82-2-920-7459, Fax: +82-2-920-2038, Email: nakyoungkim@sungshin.ac.kr

This is an Open Access article distributed under the terms of the Creative Commons Attribution Non-Commercial License (http:// creativecommons.org/licenses/by-nc/3.0) which permits unrestricted non-commercial use, distribution, and reproduction in any medium, provided the original work is properly cited.
} 
tions for gene editing is the Bioethics and Biosafety Act (hereinafter "BioAct"). The BioAct does not specify and regulate 'gene editing' itself. However, assuming that genetic editing is used in the process of research and treatment, we look to the specific details of the regulations for research on humans as well as gene therapy research in order to see how genetic editing is regulated under the BioAct.

\section{Regulation for research on gene therapy}

Article 47 of the current BioAct provides for the regulations of gene therapy under the title "Gene Therapy". The last revision of this article was on December 29, 2015. Until the last revision the contents have been changed and this revision process is highly suggestive in interpreting the regulation of current BioAct on gene therapy. Article 36(1) of BioAct 2008 specifies 'gene therapy' as the regulatory target, while $\S 47(1)$ of BioAct 2012 specifies 'research on gene therapy' as the object to be regulated. With this revision in 2008, the BioAct regulates only research on gene therapy, not gene therapy itself, in cases of (born) humans. The legislature's intentions with respect to these amendments are not clear. However, a piece of Kommentar literature, published in 2014, described that gene therapy that targets (born) human remains was still within the clinical research stage of its development (Kim et al., 2014), and stated that it is clear that clinical research is a prerequisite for treatment. Accordingly, it is possible to interpret that the scope of the research on gene therapy is the same as the scope of gene therapy. However, in order to clarify how the regulation should be applied, "Research on gene therapy" in $\S 47(1)$ of BioAct 2016 should be revised to state "Research on gene therapy and gene therapy".

\section{Uncertainty of the classification}

$\S 47$ of BioAct 2016 classifies gene therapy as: (1) procedures altering genes in the human body and (2) procedures transferring the genetic material or cells into which the genetic material has been introduced to human body.
BioAct 2012, unified the type of treatment that is the purpose of the research, without presupposing this type of classification. Whereas $\S 47(1)$ of 2012 required all gene therapy research procedures to meet requirements (1) and (2) (in which the patient must be facing a certain type of disease that threatens life or causes a severe disability and where no applicable therapy exists or gene therapy presents a greater chance of being effective than other therapies, respectively); $§ 47(2)$ of 2016 only required one of the two above requirements when the gene therapy intended to transfer genetic material (or cells into which the genetic material has been introduced) into a human body.

Unfortunately, the text of the $\S 47$ of BioAct 2016 does not make clear the kinds of activities that to which the procedures specified in type (1) and type (2) refer. The National Health and Welfare Committee's report on the revision bill states that "genetic material transfer" of type (2) is a method of delivering genetic material or cells into which the genetic material has been introduced to the human body and a method of delivering a specific substance to a cell without altering genes. In addition, the report states that this method is relatively safe and does not cause an intergenerational heritage. In contrast, the report says that the gene altering procedure of type (1) is a method that causes a genetic alteration in the human body in order to change the genetic traits. Furthermore, the report provides that altering genes raises concerns about the safety of gene therapy and suggests that intergenerational inheritance of the altered genes is likely to occur. According to this legislative explanation, the most significant difference between the type (1) and type (2) procedures is whether the act causes 'genetic alteration' in the human body and whether the result of the act is likely to be inherited intergenerationally.

Despite such an explanation, these regulations can create confusion when attempting to understand what each procedural type of gene therapy means. On the one hand, it is difficult to say that the procedure of "transferring genetic 
material or a cell into which genetic material is introduced to the human body' in $\S 47(2)$ has no possibility of altering genes in the human body if understood only in the literal sense and is therefore completely distinct from the act in $\S$ 47(1). On the other hand, $\S 47$ (2) defines the procedure by the method of applying the technology. However, the 'procedure altering genes in the human body' in $\S 47(1)$ can be made by the methods mentioned in $\S 47(2)$, i.e. by the 'invivo' or 'ex-vivo' method, apart from the result of the genetic variation. Therefore, to clear up these ambiguities the definition of gene therapy in BioAct 2016 and the description of the procedures of $\S 47(1)$ and $\S 47(2)$ need to be revised yet again.

\section{Regulation of gene scissors technology}

Understanding the definition and classification of gene therapy in BioAct 2016 does not make it clear how the provisions of the BioAct can be applied to genetic editing using gene scissors. In other words, it is not clear which type of gene therapy provided for in the BioAct 2016 corresponds to gene editing using gene scissors. Genetic editing using gene scissors can be performed in a variety of ways "depending on the type of gene scissors by which the desired site is cut, the number of gene scissors that have been delivered to the cell, or whether a donor with similar sequences has been delivered together, etc". In particular, gene therapy through genetic editing can be accomplished in a variety of ways, including: gene therapy by "removing specific genes, propagating the cells and putting the produced cells back into the patient" (ex-vivo Genome Correction by self-cell therapy) and gene therapy using a virus-based gene transfer vector to deliver specific genes (internal gene transfer therapy) (Ministry, 2015). These types of submodels seem to correspond to the transfer of genetic material or the transfer of genetic material-introduced cells, which is mentioned in gene therapy type (2) only in terms of their modes of action. However, if there is a possibility of genetic alteration in the human body using the gene scissors in this process, it corresponds to gene therapy type (1).

In legislative history related to the revision of BioAct 2016, there is no mention of whether gene scissors or genetic editing corresponds to either type (1) or type (2), or whether type (1) is specified with the gene scissors technique in mind. Among research papers that consider how gene editing can be understood in BioAct 2016, one paper emphasizes that gene editing technology is "causing genetic alteration" (Park and Ryoo, 2016). This view highlights that gene editing technology targets DNA in the nucleus and DNA in the nucleus "affects virtually every genetic feature in humans." Moreover, this paper states that the procedure of type (1) is the method for "changing the genetic traits in the human body by using gene editing technology such as Crisp scissors, etc". This interpretation implies that all forms of gene editing correspond to type (1). Even if we interpret-as the legislator mentioned-the 'possibility of genetic alteration' as 'heritability to the next generation' and interpret the type of procedure in $\S 47(2)$ as a 'simple' transmission of a genetic material or a cell into which a genetic material has been introduced, it is not always obvious that gene editing is an act corresponding to type (1).

\section{THE REGULATION OF KOREAN BIOACT (II): CASE OF EMBRYOS, OOCYTES, SPERM, AND FETUSES}

\section{A total ban on treatment}

BioAct 2016 provides that "no gene therapy [is] to be applied to an embryo, ovum, sperm,or fetus" (referred to as “embryos etc".). Following the BioAct's first introduction of gene therapy regulations, this particular regulation has been consistent. Only minor adjustments have been made, such as in BioAct 2008 in which the words, "Notwithstanding the provisions of paragraph (1)," was added to this provision. In conjunction with this gene therapy ban, the so-called gene therapy for Germline, it clearly states that BioAct strictly regulates therapy of embryos etc., as op- 
posed to providing only a limited recognition of gene therapy for (born) humans.

\section{Research on treatment}

\section{1) Uncertainty of regulation}

Article 47(3) of BioAct 2016 regulates 'gene therapy' itself with respect to embryos, etc. in contrast to regulating 'research on gene therapy' on (born) humans. Therefore, it is unclear whether this provision also prohibits gene therapy research that targets embryos and the like. As mentioned earlier, it may be interpreted that research on gene therapy targeting human embryos is also prohibited as long as gene therapy for human embryos is prohibited. However, such an interpretation is not correct in view of the regulatory system of BioAct 2016. BioAct 2016 clearly differentiates the regulation of studies of (born) humans (§ 2(1)) and embryo studies ( $\$ 29$ and below). Moreover, BioAct 2016 does not entirely prohibit research using human embryos or aimed at human embryos. Therefore, the interpretation of the permissible range of research on embryonic gene therapy is particularly controversial.

\section{2) Regulations of embryo research in BioAct}

To determine the extent to which BioAct 2016 allows gene therapy research on embryos, it is first necessary to look at how BioAct 2016 defines embryo research. Chapter 4 of BioAct 2016, "Production of Embryos, etc., and Research Thereon", provides whether embryos can be generated for research purposes, whether embryos can be used for research purposes, and to what extent these purposes are acceptable. According to $\S 23(1)$ of BioAct 2016, it is forbidden to produce embryos for the purpose of research. Therefore, it is clearly forbidden to produce embryos for the purpose of gene therapy research. However, the BioAct 2016 permits the use of "residual embryos", which remain after embryos produced as a consequence of artificial insemination are used for pregnancy, before the appearance of the primitive streak for research conducted for a specific purpose permitted by law. Furthermore, $\S$ 31(1) of BioAct 2016 permits the production of embryos through somatic-cell nuclear transplantation or parthenogenesis, as long as it is done for research purposes permitted by law. Finally, it is possible to use residual embryos, embryos produced by somatic-cell nuclear transplantation, and cells produced by parthenogenesis (parthenogenicembryos) for certain research purposes provided in $\S 23(1)$ and $\S 31(1)$. Although $\S 47(3)$ of BioAct 2016 explicitly prohibits gene therapy on embryos, these additional regulations regarding embryo research raise a controversial question over whether the use of embryos for research related to gene therapy is possible within the scope of $\S$ 23(1) and $\S 31(1)$.

One possible interpretation is that 'research on gene therapy' includes 'research on the gene therapy of a born human using embryos', considering that $\S \S 47(1)$ and (2) of BioAct 2016, unlike $\S 47(3)$, stipulate 'research on gene therapy' rather than 'gene therapy'. It is interpreted that although $\S \S 47(1)$ and (2) use the term 'human body', it is possible to conduct gene therapy research for human beings using embryos. Of course, the following arguments can be raised against this interpretation: Articles $\S \S 47(1)$ and (2) of BioAct 2016 explicitly use the term 'human body' and this provision-as mentioned before-is a comprehensive regulation of gene therapy, even if it specifies research on gene therapy.

Another possible interpretation is to emphasize that $\S \S$ 47(1) and (2) of BioAct 2016 do not clearly stipulate 'research on gene therapy using embryos' in the same context as the above counter-argument. On this basis, some interpret this to mean that research using embryos could be subject to the provisions on residual embryo studies (§ 29) and to the study of somatic-cell cloning and parthenogenic embryos (§ 31) (Jun, 2015; Kim et al., 2015a). According to this interpretation, it is possible to research gene therapy for human embryos using gene editing techniques to the 
extent that these regulations permit the research. In this interpretation, there may be varied opinions as to whether, or to what extent, research on gene therapy can be included in the scope of the research purpose set out in the regulations regarding research of residual embryos. BioAct 2016 limits the study range of the remaining embryos to: "1. Research for the development of therapies for infertility and technology for contraception", " 2 . Research on therapies for muscular dystrophy or other rare or incurable diseases specified by Presidential Decree", and "3. Research for other purposes specified by Presidential Decree through deliberation by the National Committee". Furthermore, Article 12 of the Enforcement Decree of the Bio Act (2016) specifically addresses the range of diseases for which research can be conducted in $\S 29(1)(1)-(3)$ of BioAct 2016. Therefore, some have criticized that the scope of the residual embryo research is very narrow to apply to research on gene therapy (Park and Ryoo, 2016). In other words, it seems unlikely that gene therapy research using embryos can be included within the research of specific disease treatments specified in $\S 29(1)$ of BioAct 2016.

\section{THE REGULATION OF PHARMACEUTI- CALS AFFAIRS ACT, ETC.}

Due to the fact that gene therapy products are regarded as drugs, they fall under different regulations. For instance, the Korean Pharmacopoeia Act regulates clinical trials using pharmaceuticals, and has put more stringent sanctions on clinical trials for gene therapy products. Furthermore, the Pharmaceutical Affairs Law regulates the Food and Drug Safety Directorate's ability to grant permission for the manufacture, sale, and import of pharmaceuticals. In addition, the Food and Drug Administration formulates the "Licensing and Examination Regulations for Biological Products" with a notice, and regulates the criteria for product approval. In particular, the notification regulates the criteria for licensing items for gene therapy products.

\section{Problems with approval of gene therapy products}

Article 31 of the PharmAct 2016 provides that the Food and Drug Safety Minister has the power to authorize the manufacture and marketing of pharmaceuticals. Especially, $\S 31$ (3) PharmAct 2016 provides as follows: Where a person, other than a manufacturer prescribed in paragraph (1), intends to entrust a manufacturer with the manufacture and marketing of any of the following drugs, he/she shall file a report on contract-manufacturing and marketing business with the Minister of Food and Drug Safety and obtain marketing approval for each product, as prescribed by Ordinance of the Prime Minister. Concretely, Nr. 2 of $\S 31$ (3) PharmAct 2016 specifies that the following drugs are the object of marketing approval: a biological preparation, recombinant DNA drug, cell culture technology-derived products, cellular therapy products, gene therapy products, and similar drugs for which clinical trials prescribed by Ordinance of the Prime Minister have been conducted in a foreign country in addition to the clinical trials prescribed in subparagraph (1).

Based on the provisions of the PharmAct 2016, the Food and Drug Safety Administration has enacted the "Approval and Examination Regulations for Biological Products, etc." (hereinafter referred to as "the Notification Act") as the official notification process of the Food and Drug Safety Administration. The Notification Act provides more specific criteria for approving items in the PharmAct 2016. Articles 3(2) and (3) of the Notification Act stipulates, in particular, the approval of gene therapy products. The standards set forth in $\S 3(2) \mathrm{Nr} .1$ and Nr.2 of the Notification Act are the same as those set forth in $\S \S 47(1)$ and (2) of BioAct 2016. § 3(2) of the Notification Act stipulates that gene therapy products may be approved in cases that meet one of the following conditions: 1. Gene therapy Products for a hereditary disease, Acquired Immune Deficiency Syndrome (AIDS), or any other disease that threatens one's life or causes a severe disability; 2 . Where there is no applicable therapy at present, or the safety and effica- 
cy are clearly improved compared to other therapies currently available. However, BioAct 2016 categorizes the types of gene therapy and prescribes the first and second cases (No.1 and No.2) in $\S 47(1)$ as 'and-condition' and in $\S 47(2)$ as 'or-condition'. On the other hand, the Notification Act does not yet reflect the revised contents of BioAct 2016-the conditions of $\S \S 47(1)$ and (2) of BioAct 2016 are defined as or-conditions in the Notification Act. In other words, the approval criteria for gene therapy research in the BioAct 2016 have been strengthened to meet both the disease and treatment requirements, but the Notification Act of the Food and Drug Safety Administration requires only one of the conditions of disease and treatment even after the revision of BioAct 2016. Furthermore, the Administration determines its approval for clinical trials according to its or-condition stipulation (Kim, 2015b).

However, § 3(3) of the Notification Act of the Food and Drug Safety Administration states that if the product raises an ethical concern, such as by treating genetic alteration of human germ cells, then the gene therapy product should not be approved. In other words, $\S 3(3)$ of the Notification Act does not authorize gene therapy products if genetic alteration is involved. From this perspective, $\S 3(2)$ of the Notification Act may be understood as the regulation concerning the research referred to in $\S 47(2)$ of BioAct 2016. In that case, it seems that in $\S 3(2)$ of the Notification Act it is not logically contradictory to maintain the or-condition (contrasting with the Health and Welfare Committee of the Korean National Assembly review report).

\section{Problems with clinical trials}

If gene therapy is being performed at the clinical trial level, the provisions in the PharmAct 2016 for clinical trials are also applied, apart from BioAct 2016, especially when gene therapy products are used in clinical trials. According to $\S 34(1)$ of the PharmAct 2016, each person who intends to conduct a clinical trial using drugs, etc. shall prepare a protocol thereof and obtain approval from the
Minister of Food and Drug Safety, and even where he/she intends to modify any approved matter, he/she shall obtain approval as prescribed by Ordinance of the Prime Minister. This is particularly important in the case of a clinical trial of a gene therapy product, as the Minister of Food and Drug Safety may place limits on the clinical trial, etc., which is subject to approval under paragraph (1), where such a trial is deemed likely to harm the public interest or health and sanitation (§ 34(5) PharmAct 2016). Furthermore, where any clinical trial, etc., approved under the $\S$ 34(1) of the PharmAct 2016, is conducted, in violation of any approved matter, or gives rise to serious safety and ethical issues, the Minister of Food and Drug Safety may issue an order to halt the clinical trial, to stop use of drugs for the clinical trial, to recall or abandon such drugs, or to take other necessary measures (§ 34(6) PharmAct 2016).

\section{CONCLUSION}

Finally, in case of (born) humans, the classification of the activities in $\S 47(1)$ and (2) BioAct is not clear. Furthermore, BioAct regulates research on gene therapy rather than gene therapy. Therefore, it is not easy to determine to what extent gene editing is prohibited by BioAct, even though we interpret the 'possibility of genetic alteration' as 'heritability to the next generation' and interpret the type of procedure in $\S 47(2)$ as a 'simple' transmission of a genetic material or a cell into which a genetic material has been introduced. In this context, the revision of the definition of gene therapy and the classification of gene therapy types in BioAct is needed. Moreover, in order to clarify how the regulation should be applied, "Research on gene therapy" in $\S 47(1)$ and (2) should be revised to state "Research on gene therapy and gene therapy". In case of embryos etc., gene therapy is strictly forbidden according to $\S 47(3)$ BioAct 2016. No gene therapy is allowed in case of gene therapy on embryos etc.. But $\S 47(3)$ of BioAct 2016 regulates 'gene therapy' itself with respect to embryos, etc. in 
contrast to regulating 'research on gene therapy' on (born) humans. It is contrary to the regulation of $\S 47(1)$ and (2), which stipulate the research on gene therapy. As above mentioned, one possible interpretation is that 'research on gene therapy' includes 'research on the gene therapy of a born human using embryos', considering that $\S 47(1)$ and (2) of BioAct 2016, unlike $\S 47(3)$, stipulate 'research on gene therapy' rather than 'gene therapy'. However, BioAct clearly has its own rules for research using embryos. Therefore, it is not avoidable, to apply the regulations on embryo research ( $\S 29$ and 31) to research on gene therapy using embryos. Article 12 of the Enforcement Decree specifically addresses the range of diseases for which research can be conducted in $\S 29(1)(1)$-(3) of BioAct 2016 and this scope is very narrow. Therefore, it seems very unlikely that therapy research using embryos can be included within the research of specific disease treatments specified in $\S 29(1)$ of BioAct 2016. This shows the problem of regulation on gene therapy research using embryo in Korea BioAct. Regarding the research of gene therapy, it is necessary to revise the scope of embryo research permission to reflect the trend of gene therapy research in consideration of the relationship with $\S 47$ (1) and (2). Furthermore, there exists regulations of gene therapy products as drugs. According to $\S 3(3)$ of the Notification Act based on PharmAct 2016, gene therapy products should not be authorized if genetic alteration is involved.

\section{ACKNOWLEDGEMENTS}

This work was supported by the Sungshin University Research Grant of 2017.

\section{REFERENCES}

Jun BO (2015) Ethical issues on the human embryonic genome editing. Journal of the Korean Bioethics Association 16: 17-29.

Kim HC, GO BJ, Park JS, Choi KS (2014) Bioethics Law. Pakyoungsa Press.

Kim HN, Kim SH, Kim SY (2015a) Issues of genetic modification in human embryo and its future orientation. Korean Journal of Medicine and Law 23:211-224.

Kim SK (First expert member of the Health and Welfare Committee) (2015b) Review on a revision draft of BioAct (Draft Nr.15728, Nr. 17197), November 2015.

Ministry of Science, ICT and Future Planning (2016) 2015 Technology Assessment Report (Research Report 2016048) - Vol.1. Gene Scissor Technology -

Ministry of Trade, Industry and Energy (2015) Biosafety 16(2) June 2015.

Park DW, Ryoo HS (2016) Regulatory framework for gene therapy on human embryos in response to CRISPRCas9. Journal of the Korean Bioethics Association 17: $35-52$. 